\title{
Earthquake accelerogram selection and scaling procedures for estimating the distribution of drift response
}

\author{
Nicola Buratti ${ }^{1}$; Peter J. Stafford ${ }^{2}$; Julian J. Bommer ${ }^{3}$
}

\begin{abstract}
The problem of how to select a suite of earthquake accelerograms for time-domain analyses is one of particular practical and academic interest. Research in this field has led to numerous approaches for compiling suites of accelerograms that may be used to robustly estimate the median structural response. However, many applications in earthquake engineering require the estimation of the full distribution of a structural response parameter for a particular predefined scenario. This article presents an efficient procedure whereby the distributions of inter-storey or roof drifts may be well approximated. The procedure makes use of three-point approximations to continuous distributions and the strong correlation that exists between the spectral acceleration at the initial fundamental period of the structure and the drift response. The distributions that are obtained under the proposed approach are compared to a reference distribution that is assumed to represent the true underlying distribution of drift response. The reference distribution is defined via a regression analysis conducted on the results of time-domain analyses of a six-storey reinforced concrete frame building subjected to 1666 unscaled natural accelerograms. The results indicate that robust estimates of the first and second moments of the distribution of logarithmic drift may be obtained by subjecting the structure to several accelerograms scaled to match three target spectra over a range of periods. The target spectra are defined in terms of the numbers of standard deviations above or below the median $5 \%$-damped spectral acceleration and correspond to the roots of a third-order Hermite polynomial. The results demonstrate that consideration of fifth-order Hermite polynomials does not lead to a significantly improved performance of the approach.
\end{abstract}

CE Database keywords: Ground motion; Earthquake; Dynamic structural analysis; Probability distribution; Structural reliability.

\footnotetext{
${ }^{1}$ Ph.D. Candidate, DISTART - Structural Engineering, University of Bologna, Viale Risorgimento 2, 40136 Bologna, Italy.

${ }_{2}^{2}$ RCUK Fellow / Lecturer in Modelling Engineering Risk, Willis Research Fellow, Department of Civil \& Environmental Engineering, Imperial College London, South Kensington campus, London SW7 2AZ, United Kingdom (corresponding author). E-mail: p.stafford@imperial.ac.uk

${ }^{3}$ Professor of Earthquake Risk Assessment, Department of Civil \& Environmental Engineering, Imperial College London, South Kensington campus, London SW7 2AZ, United Kingdom
} 


\section{Introduction}

For the majority of applications in which the effects of earthquakes on structures are considered, seismic actions are represented in the form of pseudo-acceleration or displacement response spectra. There are, however, many situations for which the specification of structural actions via a response spectrum is deemed insufficient. In such cases, the structural response for a given earthquake scenario is estimated by subjecting the structure to acceleration time-histories that are compatible with the scenario in question. These time-domain analyses are far more computationally expensive than response-spectrum-based alternatives but with ongoing improvements in the power of desktop computers commonly encountered in design offices, this issue is becoming less of a constraint. Recent work, such as that of Hancock et al. (2008), has demonstrated that robust estimates of the median structural response of a typical multi-degree-of-freedom (MDOF) structure may be obtained with the use of very few accelerograms if their spectra initially have an appropriate shape and the records are then scaled and adjusted with wavelets to ensure that they have particular characteristics (Hancock et al., 2006). The study of Hancock et al. (2008) is just one recent example of the numerous approaches that have been proposed for selecting, scaling and modifying real accelerograms in order to obtain robust estimates of the median structural response (e.g., Shome et al., 1998; Naeim et al., 2004; Baker and Cornell, 2006a; Watson-Lamprey and Abrahamson, 2006; Kottke and Rathje, 2008). Approaches based upon linearly scaling or wavelet adjusting real accelerograms tend to be favored now over earlier procedures that were proposed for synthesizing spectrum-compatible accelerograms (e.g., Gasparini and Vanmarcke, 1979; Boore, 2003) as these earlier approaches result in accelerograms with unrealistic energy contents (Bommer and Acevedo, 2004). Whether or not accelerograms are obtained through scaling or adjustment of real records or via synthetic means, the objective once the suite has been obtained is generally to determine a central estimate, such as the mean or median, of the structural response that may then be used for design purposes. The rationale for focusing upon a central estimate of the response is that the conditional variability of a response measure given some ground motion level is usually much smaller than the variability in the ground motion itself (Cornell, 2005). As a result, the research that has been conducted thus far has principally focused upon how to estimate the median response most efficiently as well as on how to identify the characteristics of records that lead to biased estimates of this response measure (e.g., lervolino and Cornell, 2005; Baker and Cornell, 2006a; Luco and Bazzurro, 2007; Tothong and Cornell, 2008).

There are numerous applications for which knowledge of a central estimate of the response is not sufficient and for which one requires an estimate of the full distribution of the structural response. Generally, these applications are related to the assessment of existing structures whereas the approaches focusing on estimating the median response are primarily geared towards the specification of loading for structural design (although, ultimately, knowledge of the distribution of response may have implications for the specification of design requirements in codes). For example, in earthquake loss assessment one must not only consider the potential damage associated with the 
expected response, but also the damage due to the full range of possible responses that may be experienced under a particular scenario. Likewise, when undertaking structural assessments one should consider the utility of alternative options for retrofit or demolition on the basis of costs estimated from consideration of all possible levels of future damage that might be sustained by the structure in question.

Intuitively, one would expect that more time-history analyses are required in order to estimate the characteristics of the full distribution of a particular response measure than are required to obtain an estimate of the median response. However, although some existing procedures are advertised as being capable of recovering the distribution of response measures, these procedures tend to provide this facility as a by-product of estimating the median response and the question of how many records are actually required in order to determine the response has not been directly posed (although some studies, such as that of Baker (2007), have compared the efficiency of some possible approaches). The objective of this article is to directly address this issue, without making recourse to approximate approaches such as that identified by Shome et al. (1998), and to assess whether it is feasible to obtain robust estimates of the distribution of inter-storey drift in a design-office situation. As a result, we outline an efficient approach via which the full distribution of drift response may be approximated using estimates of the first two moments of the distribution. A key assumption of this approach, that is tested in the article, is that the drift values, conditioned on some target spectral acceleration level, are lognormally distributed (hence, knowledge of the first two moments fully defines the distribution). While the numbers of records that are required under this approach are greater than that required to estimate the median alone, it is shown that the numbers of records are not as high as one might imagine and that it is plausible to run these analyses in a design-office environment.

\section{Overview of the procedure}

The objective of the research presented herein is to identify methods via which the distribution of drift response may be estimated for a given seismological scenario. In order to evaluate the performance of any approach one must first establish a basis for comparison. In the present case the true distribution of drift response corresponding to any particular scenario is unknown and we must therefore define some reference distribution that may be adopted as a surrogate for the true, but ultimately unknowable, distribution of drift. To this end the approach adopted by Hancock et al. (2008) is implemented whereby a structure is analyzed under the action of a very large number of unscaled natural accelerograms. The drift responses that are obtained during these analyses are then compiled and empirical models that relate the calculated drift values to common seismological parameters are derived via regression analysis. An empirical model for estimating spectral accelerations over a broad range of periods is also derived. Given these models one may take an earthquake scenario, defined during a seismic hazard analysis, and obtain the distribution of spectral acceleration or drift values that are associated with this scenario. Provided that the dataset used for the development of the 
empirical models is large and that the seismological scenario being considered is not beyond or near the magnitude-distance range of applicability of the models (Bommer et al. 2007), it is reasonable to assume that the distributions thus obtained will be good approximations to the true, but unknown, distributions. Although this assumption cannot be validated, most empirically-based methods within engineering seismology and earthquake engineering are founded upon similar assumptions.

A fundamental assumption of the regression analyses that have been conducted, and that are presented herein, is that the ordinates of spectral acceleration and the inter-storey and roof drifts are lognormally distributed. The assumption of lognormality of spectral acceleration is ubiquitous in earthquake engineering and has recently been rigorously tested by Jayaram and Baker (2008). This assumption is also well supported by the distributions of residuals that were obtained following the regression analyses. The assumption regarding the lognormality of the drift values has received far less attention, although some cursory analyses have been made (Shome et al., 1998) and the assumption is embodied within common practice (Cornell et al., 2002). The assumption of lognormality of drift response is of particular importance in the present study as by assuming a lognormal distribution during the regression analysis we are also making an implicit assumption regarding the nature of the reference distribution. For the purposes of the current study, making the assumption that the drift values are lognormally distributed is also very convenient as it means that the distribution of drift may be fully described by just the first and second moments of the distribution, i.e., if one can obtain robust estimates of the mean and standard deviation of the logarithmic drift then the complete distribution is known. Given the pivotal importance of this assumption to the approach advocated herein, formal statistical tests are conducted upon the residuals obtained from the regression analyses; the results of these tests are presented later in the article.

The large number of time-history analyses that are required for deriving the empirical relationships, and hence the reference distributions, may also be used to infer relationships among the ground-motion parameters and measures of structural response. For example, if one plots the calculated inter-storey drifts against the spectral accelerations at the fundamental period of the structure one is able to observe very strong dependencies (Figure 1). Such dependencies are useful for identifying functional relationships among different parameters such as spectral acceleration and roof drift, and the existence of such trends implies that a robust model for the median drift may be obtained from the median spectral acceleration. For the purposes of the present study, however, we are more concerned with how the spectral acceleration and drift values are distributed about these median values. In particular if we are able to demonstrate that a higher-than-average, or positive epsilon (Baker and Cornell, 2006a), spectral acceleration also leads to a higher-than-average drift in a systematic manner then we may be well placed to relate the distribution of drift values to the distribution of spectral acceleration values. 


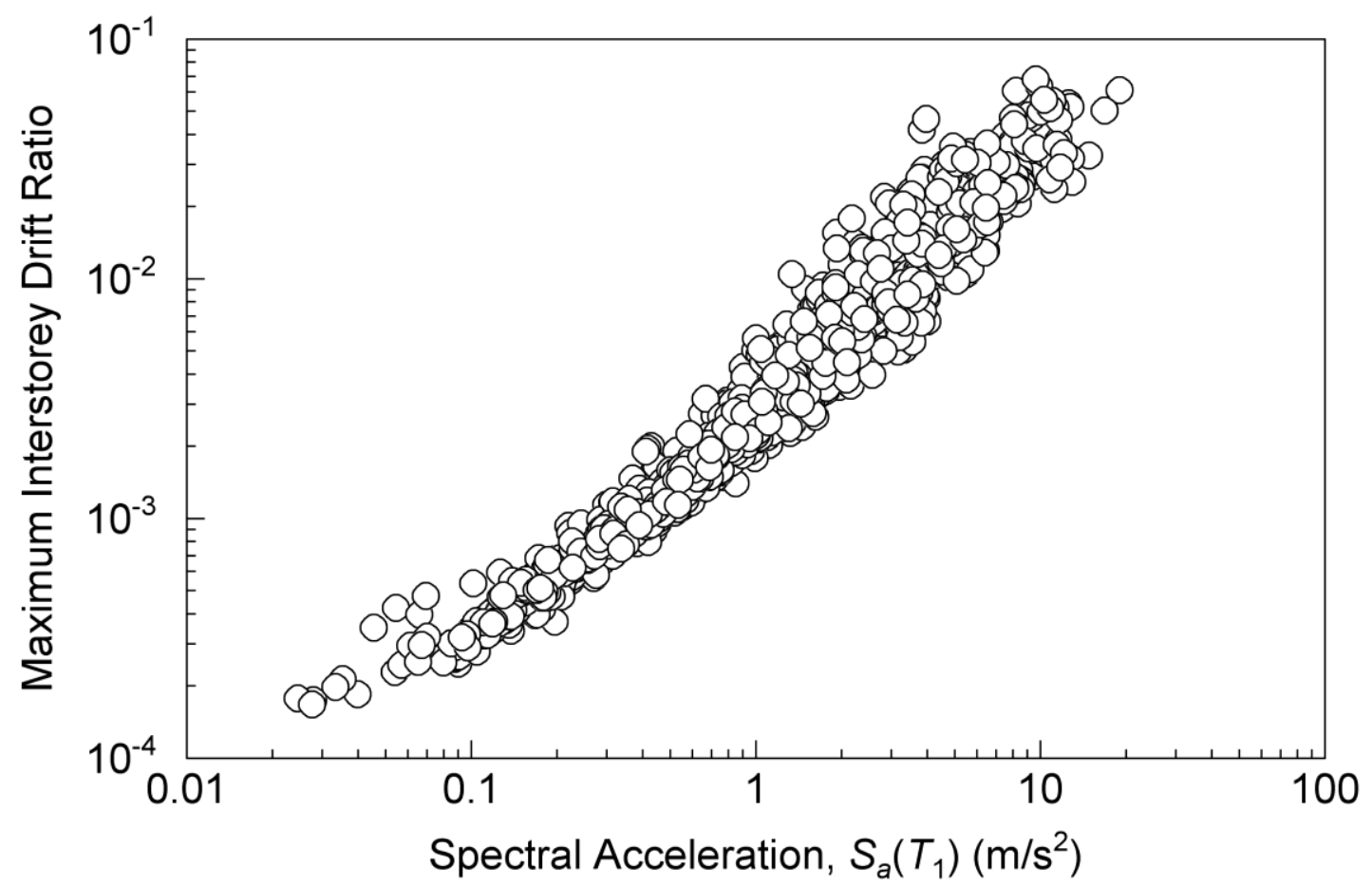

Figure 1: Relationship between maximum inter-storey drift and the spectral acceleration at the fundamental period of the structure, $S_{a}\left(T_{1}\right)$, found from the 1666 time-domain analyses conducted in this study using unscaled natural records.

The above reasoning forms the basis of the approach taken in this study. First a large number of time-history analyses are conducted and the results of these analyses are used to derive empirical models for inter-storey and roof drift. These models are then analyzed in order to look for correlations that exist among the residuals of the models for the drifts and the residuals of the model for spectral acceleration at the initial fundamental period of the structure. If strong correlations exist then it is possible to relate particular levels of spectral acceleration to particular levels of drift response. Hence, the rationale is that if one linearly scales a record to a particular level above or below the median spectral acceleration for a given scenario, then one may anticipate that the drift response will also be above or below the median drift response to a similar degree, as measured in units of standard deviation (assuming a positive correlation in this case). If such inferences are possible then all that remains is to identify the optimal way of mapping levels of spectral acceleration into levels of drift and hence recovering the distribution of drift. Note that an additional caveat must be made here. It is also important to ensure that the scaled records retain an appropriate spectral shape. It is well known (Baker and Cornell, 2006a; Luco and Bazzurro, 2007) that the spectral shape away from the fundamental period of a structure has a strong influence upon nonlinear response. The scaling that is performed in this study is done to target spectra rather than individual spectral ordinates, such as $S_{a}\left(T_{1}\right)$, and this means that higher-than-average spectral accelerations will lead to higher-than-average drift responses.

Two different approaches are taken for mapping the distribution of logarithmic spectral acceleration into the distribution of logarithmic drift. It must be noted that although two approaches are investigated herein, there are many other alternative approaches for 
representing a continuous distribution by a discrete approximation (Keefer and Bodily, 1983). The first approach that is taken is to subdivide the realm of possible logarithmic spectral acceleration values into ranges that have an equal probability of occurring. This is achieved by subdividing the range of cumulative probabilities into equal intervals and then using the inverse cumulative distribution function to obtain the logarithmic spectral acceleration values that correspond to the centers of these probability intervals (note that this is similar to the 'centroid method' of Kottke and Rathje, 2008). The logarithmic spectral acceleration values are identified by their corresponding epsilon values, i.e., the number of standard deviations above or below the median value. This approach is demonstrated in the left and center panels of Figure 2, where three- and five-point approximations to the standard normal distribution are shown. Note that although the ordinate is subdivided into equal intervals the resulting epsilon values on the abscissa are not equally spaced for fourpoint approximations and higher. This method of approximating a continuous distribution is rather common due to its simplicity (Keefer and Bodily, 1983; Miller and Rice, 1983) but it is known to have some deficiencies. For example, Miller and Rice (1983) demonstrate that while the mean value of both the cumulative and discrete distributions will be the same, the even moments of the discrete distribution should be less than those of the continuous distribution. The second approach adopted is more sophisticated and makes use of an approximation based upon Gauss-Hermite quadrature. Miller and Rice (1983) have demonstrated that the moments of a continuous distribution may be exactly replicated by a discrete distribution consisting of epsilon values (nodes) and associated probabilities (weights) that correspond to the nodes and weights used in Gauss-Hermite quadrature. The $n^{\text {th }}$ order Hermite polynomial may be defined as (Spiegel and Liu, 1999):

$$
H_{n}(x)=(-1)^{n} e^{x^{2} / 2} \frac{d^{n}}{d x^{n}} e^{-x^{2} / 2},
$$

which leads to the following expressions for the third- and fifth-order polynomials:

$$
H_{3}(x)=x^{3}-3 x \text { and } H_{5}(x)=x^{5}-10 x^{3}+15 x
$$

These polynomials along with their roots are plotted in Figure 2. The roots of these polynomials correspond to the epsilon values that should be used to define target levels for scaling the accelerograms. The results that are obtained from the structural analyses conducted using records scaled according to these epsilon values are then combined with the corresponding weights to obtain estimates of the moments of the distribution. The moments of the logarithmic drift may be expressed as:

$$
\left\langle\left(\log _{10} \delta\right)^{k}\right\rangle=\sum_{i=1}^{N} p_{i}\left(\log _{10} \delta_{i}\right)^{k} \quad \text { for } \quad k=0,1,2, \ldots
$$

where $\left\langle\left(\log _{10} \delta\right)^{k}\right\rangle$ is the $k^{\text {th }}$ moment of $\log _{10} \delta$, i.e., the mean and variance of $\log _{10} \delta$ correspond to $k=1$ and $k=2$ respectively, and $p_{i}$ are the weights. 

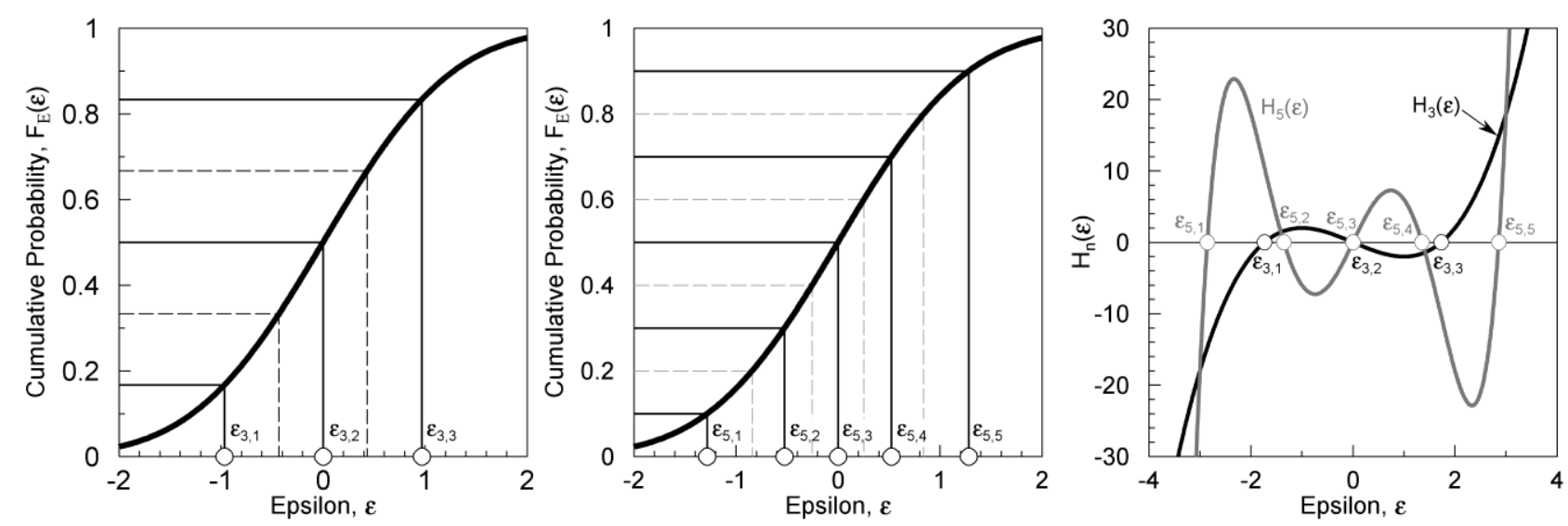

Figure 2: Left, three levels of epsilon found from subdividing the possible range of cumulative probability into equal intervals and taking the abscissa corresponding to the central value of each

interval; center, as for the left panel but for five levels. Right, third- and fifth-order Hermite polynomials and their corresponding roots. For all panels the open circles represent the epsilon values that will later serve as target levels for scaling accelerograms.

From a statistical point of view this second approach should perform better than the simpler first approach as the moments obtained via Equation 3 using the discrete nodes and weights are identical to the moments of the equivalent continuous distribution (Miller and Rice, 1983). Figure 2 presents examples corresponding to three- and five-point approximations to normal distributions and these approximations are those that we opt to use for the remainder of the analysis. However, it should be noted that there is no particular reason for having chosen to use three- and five-point approximations. Whether or not superior performance may be achieved using alternative orders for the polynomials remains to be tested.

Once the epsilon values have been defined, these may be used in conjunction with the empirical model for spectral acceleration in order to define either three or five target spectra that correspond to a particular seismological scenario. Note that in this study we follow the common assumption that the spectral ordinates are fully correlated, i.e., for a given target spectrum the ordinates at every period are the same number of standard deviations away from the median spectral level. The scaled accelerograms whose spectra best match these multiple target spectra, as quantified by the root-mean-square difference between the logarithmic target and candidate spectra over a range of periods, are then used as inputs into time-history analyses and the drift responses of the structure under these records are obtained. The statistics of the drift values are then compared to the statistics of the reference distribution defined by the empirical models derived from the time-history analyses conducted on the unscaled accelerograms.

\section{Case study}

The principle of the procedure is very simple but it relies heavily on the assumption that the characteristics of ground-motions that lead to high spectral acceleration values also lead to high drift values. In order to test this assumption an example is necessarily 
required. The particular application of the proposed procedure is outlined in the following sections.

\section{Structural model and nonlinear analysis}

A six-storey, three-span reinforced-concrete frame building is considered in this study. The geometry and reinforcement details of the planar 2D frame are shown in Figure 3. This building was designed according to the regulations given by the Italian Building Code for structures located in seismic zone 1. The area and placement of reinforcement in the individual members are also shown. The structure has an initial fundamental period of 0.93 seconds and the periods of the second to fourth mode are $0.32,0.17$ and 0.12 seconds respectively. The modal participation factors for these four modes are $0.80,0.13,0.04$ and 0.01 indicating that the structure is first-mode dominated. Pushover analyses were conducted for the columns with the resulting curves shown in Figure 4. The time-domain analyses take into account both geometric nonlinearity and material inelasticity. Structural members are modeled using force-based fiber elements (Spacone et al. 1996) which have the advantage over displacement-based approaches in that they satisfy equilibrium in the nonlinear range of material response, alleviating the need for mesh refinement. The confined concrete constitutive behavior is modeled using the theoretical model proposed by Mander et al. (1988), the unconfined concrete constitutive behavior is modeled using the model developed by Saenz (Ceb-Fip, 1993) and the steel constitutive behavior is modeled using the Menegotto-Pinto (1973) model. All nonlinear time-history analyses were conducted using OpenSees (McKenna et al. 2000).

\section{Dataset}

Accelerograms were selected from a subset of the records in the Next Generation of Attenuation (NGA) project database (Power et al. 2006). A total of 1666 accelerograms were used for the analysis. This subset was obtained by excluding all records from the Chi-Chi sequence, as well as any records with only one horizontal component and records for which appropriate metadata were not available $\left(M_{w}, R_{J B}\right.$ and $\left.V_{S, 30}\right)$. The exclusion of records from the Chi-Chi sequence was done to prevent any single earthquake from dominating the dataset. All of the selected records have a maximum usable period of at least 3 seconds (Boore and Bommer, 2005). There is a significant degree of overlap between the dataset used in the present study and that used by Hancock et al. (2008); the interested reader is referred to that article where a plot of the magnitude-distance distribution of the dataset may be seen.

\section{Regression Analyses}

For reasons of economy the same functional form was used to model both the spectral accelerations and the various measures of drift (both inter-storey and roof drifts) that are considered. The functional form adopted in this study is similar to that previously used by Hancock et al. (2008) but with a key difference being the inclusion of the average shearwave velocity over the uppermost $30 \mathrm{~m}$ at the site as an additional predictor variable in 
place of dummy variables for site classes. The functional form adopted for this study also omits the previously included dummy variables for style-of-faulting as these were not found to be statistically significant during the regression analysis (and have very little impact upon spectral shape).

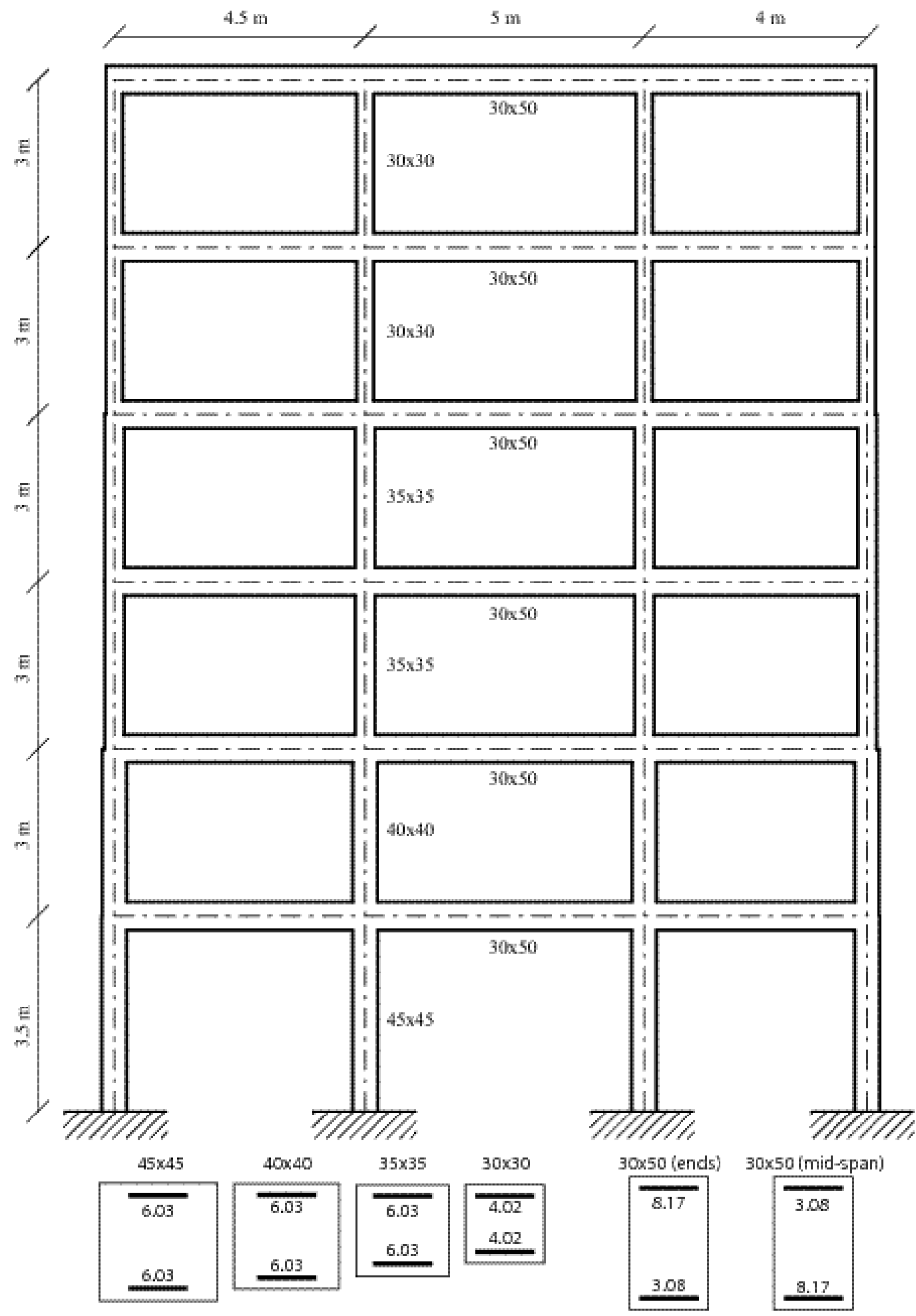

Figure 3: Geometry and reinforcement details of the 2D reinforced concrete frame considered in this study. Reinforcement details have units of $\mathrm{cm}^{2}$. 


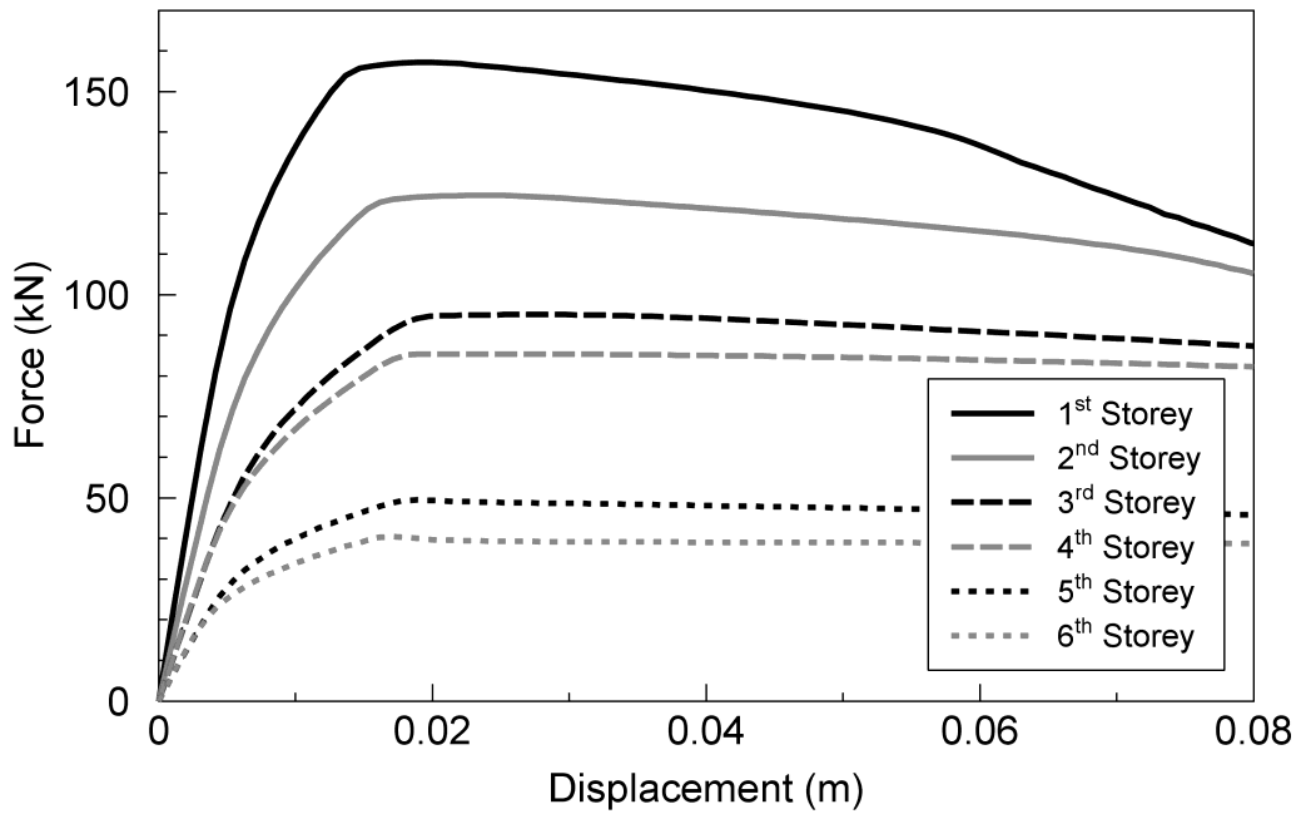

Figure 4: Pushover curves for the columns of the frame structure. The compressive loads correspond to the gravity loads relevant to the individual columns.

The functional form may be seen in Equation 4:

$$
\log _{10}(y)=c_{1}+c_{2} M_{w}+c_{3} M_{w}^{2}+\left(c_{4}+c_{5} M_{w}\right) \log _{10}\left(\sqrt{R_{J B}^{2}+c_{6}^{2}}\right)+c_{7} \log _{10}\left(V_{S, 30}\right),
$$

where $y$ is the quantity for which an empirical model is desired (either a spectral acceleration or a measure of drift), $c_{1, \ldots, 7}$ are the coefficients of the model to be estimated via the regression analysis, $M_{w}$ is the moment magnitude, $R_{J B}$ is the closest distance to the surface projection of the fault rupture, as defined by Joyner and Boore (1981), and $V_{S, 30}$ is the average shear-wave velocity over the uppermost $30 \mathrm{~m}$ at the site. The total standard deviation, $\sigma_{T}$, of each empirical model may be decomposed into three independent components: the inter-event standard deviation, $\sigma_{E}$; the intra-event standard deviation, $\sigma_{A}$; and the inter-component standard deviation, $\sigma_{C}$, which must be considered when both horizontal components of a ground-motion recording are used in the regression analysis, as is done in this study (Boore et al. 1997, 2005; Baker and Cornell, 2006b). As these three components of variability are independent, the total standard deviation may be represented as in Equation 5.

$$
\sigma_{T}=\sqrt{\sigma_{E}^{2}+\sigma_{A}^{2}+\sigma_{C}^{2}}
$$

The coefficients and the variance components of the models were obtained using the nonlinear mixed effects procedure of Lindstrom and Bates (1990) as implemented in the statistical computing package R (Pinheiro et al. 2008; R Development Core Team, 2008). As in the study of Hancock et al. (2008), the regression parameters that have been derived are not presented here as they relate directly to the particular frame structure used herein and are not relevant to other applications. 
As mentioned earlier, a fundamental assumption that is made when assuming a functional form such as that in Equation 4 is that the dependent variable, $y$, is lognormally distributed (when conventional regression techniques are then used). The assumption that the spectral acceleration values are lognormally distributed has been tested recently (Jayaram and Baker, 2008) but formal tests for the lognormality of the drift values has not received such attention. Given the importance of this assumption to the approach taken in this study, statistical tests for normality were carried out on the residuals (both inter-event and intra-event) arising from the regression analyses. Numerous tests for normality are available in the statistical literature, here we have selected the Anderson-Darling test (Anderson and Darling, 1952), the Kolmogorov-Smirnov test (Massey, 1951), the Lilliefors test (Lilliefors, 1967), and the Shapiro-Wilks test (Shapiro and Wilk, 1965). Table 1 presents the $p$-values (to be interpreted as the probability that the observed residuals would have been obtained if the parent distribution was actually normal) obtained from these four tests. If a p-value of less than 0.05 is encountered then we state that evidence exists against the assumption that the drift values are lognormally distributed. As can be seen from Table 1, it appears as though the assumption of lognormality is not strong for the intra-event residuals for the fifth storey and that the test results are inconclusive for the intra-event residuals for the sixth storey. However, for all other storeys, and the roof drift, the statistical tests suggest that the assumption of lognormality of the drift values is a very strong one. It should be noted that these test statistics do not allow one to state that the drifts are lognormally distributed, what they say is that there is insufficient evidence to state that they are not lognormally distributed. With these test results in mind, we proceed under the, now justified, assumption that estimates of the mean and variance of the logarithmic drift values are sufficient to define the overall distribution of inter-storey drift.

Table 1: $p$-values for the statistical tests for normality conducted upon the considered drift measures. A-D = Anderson-Darling; $K-S$ = Kolmogorov-Smirnov; $L=$ Lilliefors; and S-W = ShapiroWilks tests. Bold text indicates values less than 0.05 .

\begin{tabular}{|c|c|c|c|c|c|c|c|c|}
\hline & \multicolumn{4}{|c|}{ Inter-event residuals } & \multicolumn{3}{c|}{ Intra-event residuals } \\
\hline & A-D & K-S & L & S-W & A-D & K-S & L & S-W \\
\hline$\delta_{1}$ & 0.899 & 0.974 & 0.787 & 0.703 & 0.298 & 0.222 & 0.107 & 0.192 \\
\hline$\delta_{2}$ & 0.704 & 0.928 & 0.741 & 0.570 & 0.543 & 0.400 & 0.479 & 0.114 \\
\hline$\delta_{3}$ & 0.592 & 0.903 & 0.890 & 0.573 & 0.329 & 0.374 & 0.287 & 0.150 \\
\hline$\delta_{4}$ & 0.650 & 0.851 & 0.805 & 0.624 & 0.080 & 0.281 & $\mathbf{0 . 0 2 7}$ & 0.131 \\
\hline$\delta_{5}$ & 0.539 & 0.778 & 0.754 & 0.456 & $\mathbf{0 . 0 0 1}$ & $\mathbf{0 . 0 3 5}$ & $\mathbf{0 . 0 0 1}$ & $\mathbf{0 . 0 0 0}$ \\
\hline$\delta_{6}$ & 0.293 & 0.696 & 0.786 & 0.237 & $\mathbf{0 . 0 0 0}$ & 0.093 & $\mathbf{0 . 0 3 1}$ & $\mathbf{0 . 0 0 0}$ \\
\hline$\delta_{\text {roof }}$ & 0.675 & 0.922 & 0.926 & 0.568 & 0.088 & 0.312 & 0.085 & 0.132 \\
\hline
\end{tabular}




\section{Correlations between $S_{a}\left(T_{1}\right)$ and $\delta_{i}$}

Following the development of the empirical models, the residuals were examined in order to identify the strength of any correlations that exist between the spectral acceleration at the initial fundamental period and the drift values. For the purpose of determining the correlations it is important to make the distinction between the inter-event and intra-event components of the total residuals. The results indicate that correlations between all of the considered drift measures (the six inter-storey drifts and the roof drift) and the spectral acceleration at the initial fundamental period of the structure are very strong. The strength of these correlations may be appreciated by inspection of Table 2 where all of the calculated correlations are presented, as well as in Figure 5 where examples of the normalized residuals are shown (see Stafford et al. (2008) for details on how such residuals may be computed). The strength of these correlations strongly suggests that if one were to scale an accelerogram to a level of, say, one epsilon, then one should expect that the drift values that are obtained following a time-history analysis conducted with this scaled record will also be very close to a one epsilon (i.e., $84^{\text {th }}$-percentile) level in terms of drift.
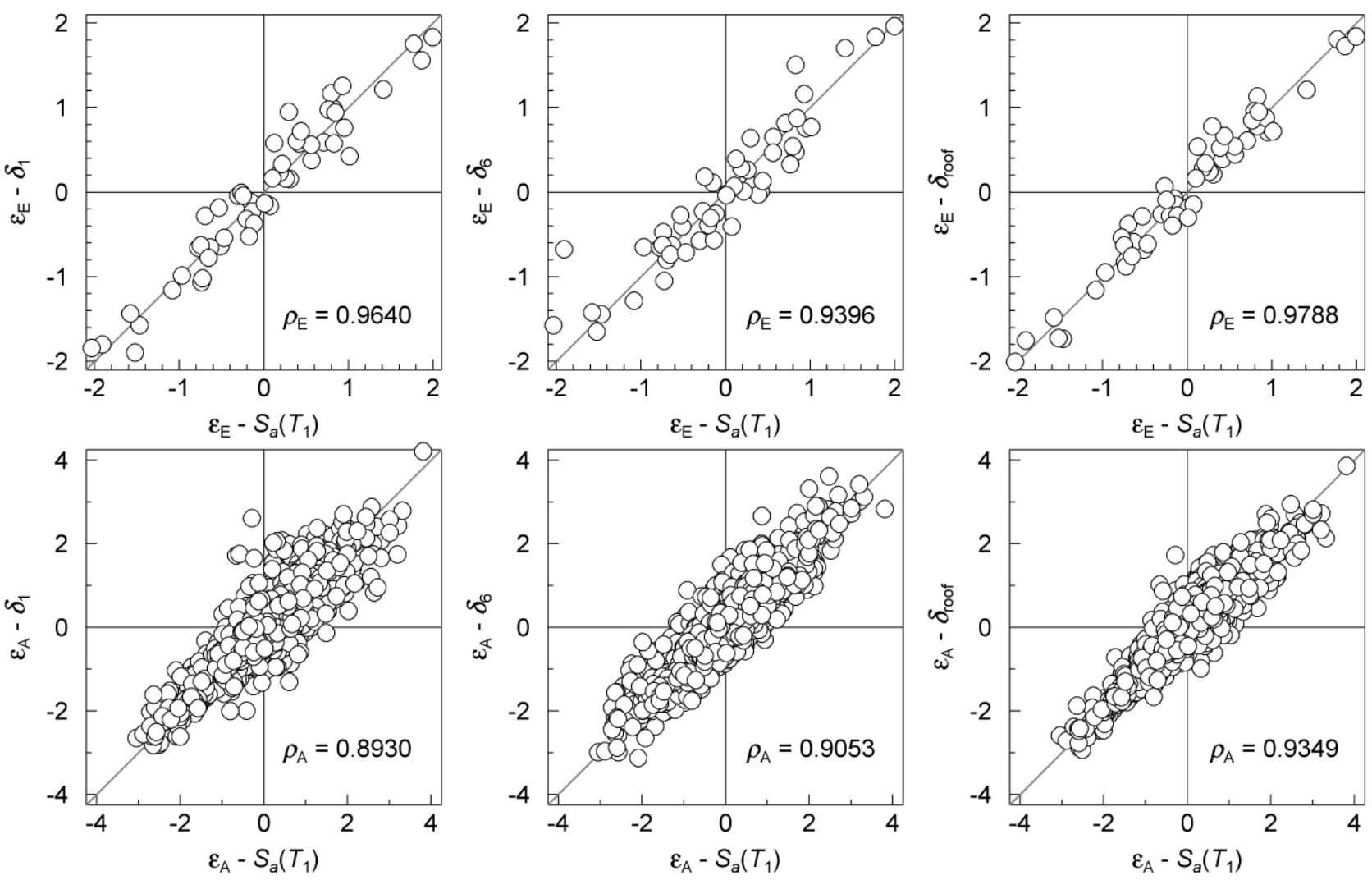

Figure 5: Correlation between the spectral acceleration at the initial fundamental period of the structure and various drift measures. The top row shows the correlation among normalized interevent residuals, $\varepsilon_{E}$, while the bottom row shows the correlation among the normalized intra-event residuals, $\varepsilon_{A}$. The plots correspond to the inter-storey drift of the first floor (left), sixth floor (middle) and the roof drift (right). The gray diagonal line represents the 1:1 equivalence. 
Table 2: Correlations between normalized inter-event, intra-event and total residuals for spectral acceleration at the fundamental period of the structure with the corresponding normalized residuals for the seven considered drift measures.

\begin{tabular}{|c|c|c|c|c|c|c|c|}
\hline $\begin{array}{c}\text { Correlation } \\
\text { with } \mathrm{S}_{\mathrm{a}}\left(\mathrm{T}_{1}\right)\end{array}$ & $\begin{array}{c}1^{\text {st }} \text { Storey } \\
\text { Drift, } \delta_{1}\end{array}$ & $\begin{array}{c}2^{\text {nd }} \text { Storey } \\
\text { Drift, } \delta_{2}\end{array}$ & $\begin{array}{c}3^{\text {rd }} \text { Storey } \\
\text { Drift, } \delta_{3}\end{array}$ & $\begin{array}{c}4^{\text {th }} \text { Storey } \\
\text { Drift, } \delta_{4}\end{array}$ & $\begin{array}{c}5^{\text {th }} \text { Storey } \\
\text { Drift, } \delta_{5}\end{array}$ & $\begin{array}{c}6^{\text {th }} \text { Storey } \\
\text { Drift, } \delta_{6}\end{array}$ & $\begin{array}{c}\text { Roof } \\
\text { Drift, } \delta_{\text {roof }}\end{array}$ \\
\hline Inter-event & 0.9640 & 0.9709 & 0.9742 & 0.9814 & 0.9615 & 0.9396 & 0.9788 \\
\hline Intra-event & 0.8930 & 0.9126 & 0.9240 & 0.9357 & 0.9250 & 0.9053 & 0.9349 \\
\hline Total & 0.9177 & 0.9327 & 0.9416 & 0.9515 & 0.9387 & 0.9224 & 0.9502 \\
\hline
\end{tabular}

\section{Record Selection}

Prior to describing the proposed approach to record selection it is necessary to define the seismological scenario that was considered for this case study. The scenario corresponds to an event with a moment magnitude of $M_{w} 7.0$ located at a distance of $R_{J B}=10 \mathrm{~km}$ from a site with an average shear-wave velocity over the uppermost $30 \mathrm{~m}$ of $V_{S, 30}=300 \mathrm{~m} / \mathrm{s}$. This scenario is purely hypothetical and was not selected with a view to representing any particular situation. However, the scenario was also not defined arbitrarily. The primary reason for working with this scenario is that it represents a severe loading case that should ensure that the structure behaves in a nonlinear manner. This combination of magnitude, distance and site class is also one for which a reasonable number of records exist, which thus provides one with more options when implementing the adopted selection procedure outlined in what follows. The final key reason is associated with the confidence that we may place in the empirical models that have been derived for the drift responses. Given that there are a good number of records that have seismological characteristics similar to those of the specified scenario and that the scenario is well within the range of parameter values used to derive the empirical models, we can be reasonably confident that the model will perform robustly and will act as a good surrogate for the unknown true distribution of drift. Hence, we can be confident that any biases that we observe in the final results are predominantly due to real biases rather than being due to a poor definition of the basis for comparison.

Numerous researchers have demonstrated, either explicitly (e.g., Hancock et al. 2008) or implicitly (e.g., Baker and Cornell, 2006a; Luco and Bazzurro, 2007), that an effective way to prevent biased estimates of structural response from occurring when using scaled records is to ensure that careful attention is paid to the spectral shapes of the accelerograms. That is, it is important to ensure that when accelerograms are scaled their spectra match a target spectrum at multiple periods so that one does not inadvertently select records that contain significant peaks or troughs at particular periods. As our sole focus in this study is upon estimating the distribution of drift response, selection on the basis of spectral shape should take priority over other commonly imposed constraints such as restrictions on the magnitude of the event from which the original accelerogram came. This prioritization is justified as Hancock and Bommer (2006; 2007) have demonstrated that peak responses like drift are not particularly sensitive to the duration of shaking. That said, when selecting records for the purpose of estimating the structural response due to a particular seismological scenario it is, in principle, preferable to select records that are at 
least broadly consistent with this scenario and for this reason an initial screening was made to limit the selection of accelerograms to those coming from events having magnitudes within \pm 0.2 units of the specified scenario. Following this initial screening, records were selected on the basis of their ability to fit, when scaled, the target spectra over a period band ranging from just below the period corresponding to the second-mode of the structure to roughly double the initial fundamental period of the structure, i.e., over the range $[0.2,2.0]$ seconds. The goodness-of-fit is quantified by the root-mean-square difference $\left(D_{r m s}\right)$ between the logarithmic ordinates of the candidate spectrum, $S a_{R}$, and the logarithmic ordinates of the target spectrum, $S a_{T}$, in the interval $\left[T_{a}, T_{b}\right]$ :

$$
D_{r m s}(\alpha)=\sqrt{\frac{1}{a-b+1} \sum_{i=a}^{b}\left(\log _{10}\left[\alpha \cdot S a_{R}\left(T_{i}\right)\right]-\log _{10}\left[S a_{T}\left(T_{i}\right)\right]\right)^{2}}
$$

where $\alpha$ is a scaling factor. For this particular study, the $D_{r m s}$ values were evaluated using a set of 36 periods, that are approximately logarithmically spaced and, that correspond to the periods used in the Caltech Blue Book system (Brady et al., 1973) within the range $[0.2,2.0]$ seconds. For each candidate record the scaling factors that minimize the $D_{r m s}$ are calculated for each of the different target spectral levels. The records that are finally selected are those with the smallest $D_{\text {rms }}$ after scaling (Beyer and Bommer, 2007). The boundaries of the period range are defined following consideration of the framework of direct displacement-based design (Priestley et al. 2007).

The selection procedure outlined above is approached in two ways. In the first approach, each of the three or five target spectral levels (prescribed either via the equi-probability, EP, approach or the Gauss-Hermite, GH, approach) are considered independently and the records whose scaled spectra best fit each of these levels are selected. In this first approach the selection is performed without replacement so that once a record has been selected it is no longer a candidate for selection at any of the other target levels. In the second approach, all target levels are considered simultaneously and the selected records are those whose spectra are able to be scaled (by three or five different factors as the case may be) in order to provide a good match to all target levels. Initially only the first approach was considered but it was observed that the standard deviation of the empirical model for spectral acceleration is fairly constant across periods with the implication (when specifying target ordinates using the same epsilon over all periods) that the shapes of the target spectra are all quite similar. Hence, if a record has a spectrum that matches one level it will also have a reasonable fit to the other levels and one may therefore work with a reduced set of accelerograms. Note that this does not imply a reduction in the number of structural analyses, just a smaller number of accelerograms that must then be scaled multiple times and that the constraint of non-replacement following selection becomes redundant. Figure 6 demonstrates the results of this second selection procedure for the four different methods of specifying target spectral levels considered in this study. Note that in each case the target spectrum corresponding to $\varepsilon=0$ is the same, yet different accelerograms are chosen due to their ability to match all of the target levels simultaneously. 

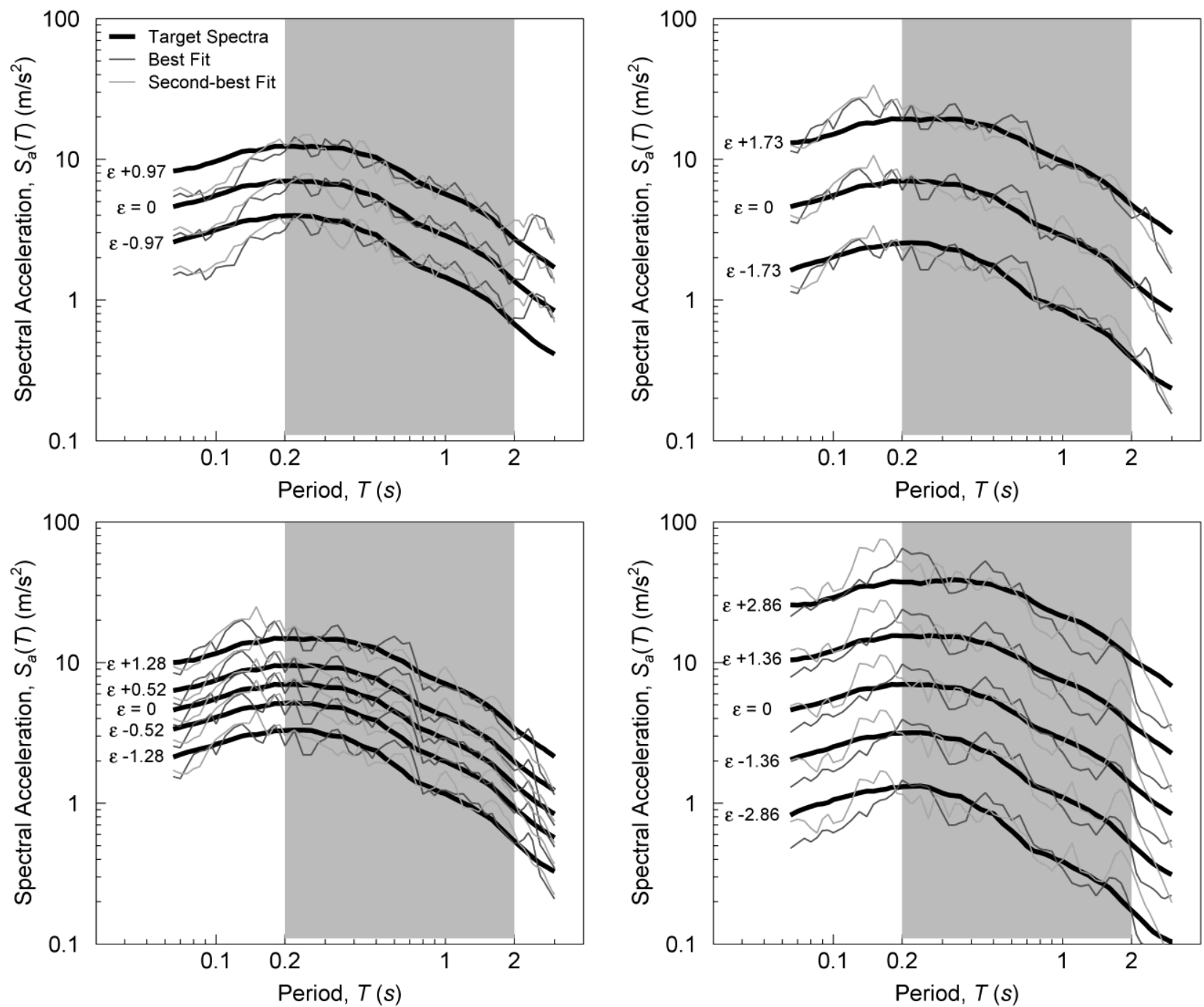

Figure 6: Examples of the spectra corresponding to the best and second-best fits (gray lines) to the target spectra (heavy black lines). Top left, three-point equi-probability approach; bottom left, fivepoint equi-probability approach; top right, three-point Gauss-Hermite approach; bottom right, fivepoint Gauss-Hermite approach. The annotated epsilon values correspond to the nodes shown in Figure 1. In all panels the shaded region represents the period range (0.2-2.0 seconds) over which the fit was evaluated.

\section{Results}

For both the EP and GH approaches the procedure based upon 3-point and 5-point approximations was evaluated for all inter-storey drifts and the roof drift. Previously, in Table 2 it was observed that the weakest correlation determined from the analyses of the unscaled records was between the spectral acceleration and the first-storey drift (although these parameters were still strongly correlated) while the strongest correlations were found to exist between the spectral acceleration and the fourth-storey drift followed very closely by the roof drift. The underlying assumptions of the methodology proposed herein would suggest that the worst performance of the method should be observed for the first-storey drift while the best performance should be observed for the fourth-storey and roof drifts. For this reason we present the results of the analyses conducted on the scaled records for 
the first-storey drift in Figure 7 and the roof drift in Figure 8. For all of the other measures of drift, for which the results are not presented herein, the performance of the approaches exhibited similar trends and the results shown in Figures 7 and 8 may be regarded as being indicative of the general performance of the approaches for this structure.

In both Figures 7 and 8 the estimates of the first two moments are shown in addition to the $95 \%$ confidence interval about this estimate. It is very clear that the performance of the Gauss-Hermite approach is significantly superior to that of the equi-probability approach, although it is important to note that different scales have been used on the ordinates of these figures. In the vast majority of cases it may be appreciated that the $95 \%$ confidence interval includes the reference level that we are assuming to represent the true value of either the mean logarithmic drift or the standard deviation of the logarithmic drift. This implies that in most cases we cannot state that the estimates based upon the scaled analyses are significantly different (in a statistical sense) from the reference levels shown in these figures by the heavy gray lines.

It is conceivable that readers may find the strong performance of these simple methods somewhat surprising and suspect that the very strong correlations that have been observed in Figure 5 are being heavily dominated by responses within the linear elastic range (i.e., Figure 1). However, we must emphasize that this is not the case. Tables 3 and 4 show the maximum drift demands experienced by the columns of the various storeys under the action of the records selected for the considered scenario. These values are presented as drift demands but are not normalized by the column height in order to enable direct comparisons to be made with the pushover curves shown in Figure 4. It is clear that the structure is experiencing significant nonlinear response and that the results that have been obtained are not simply relevant for situations in which linear, or weakly nonlinear, response is to be expected. The primary reason why the approach is able to perform well for nonlinear ranges of response, when the actual fundamental period of the structure has elongated, is that due account has been taken of the spectral shape. Hence, the strength of the correlation among the drift values and the spectral acceleration at the elongated period of the structure should be effectively preserved.

Table 3: Record selection based upon single records scaled to multiple reference spectra. The numbers in the table represent the maximum drift demands in meters, i.e., directly comparable to the pushover curves shown in Figure 4. GH = Gauss-Hermite, EP = Equal Probability.

\begin{tabular}{|c|c|c|c|c|c|c|}
\hline & \multicolumn{7}{|c|}{ Storey } \\
\hline Method & $1^{\text {st }}$ & $2^{\text {nd }}$ & $3^{\text {rd }}$ & $4^{\text {th }}$ & $5^{\text {th }}$ & $6^{\text {th }}$ \\
\hline GH 3 levels & 0.133 & 0.111 & 0.111 & 0.087 & 0.192 & 0.144 \\
\hline GH 5 levels & 0.2555 & 0.249 & 0.18 & 0.096 & 0.75 & 0.192 \\
\hline EP 3 levels & 0.0735 & 0.063 & 0.066 & 0.045 & 0.15 & 0.09 \\
\hline EP 5 levels & 0.1155 & 0.096 & 0.105 & 0.066 & 0.174 & 0.114 \\
\hline
\end{tabular}



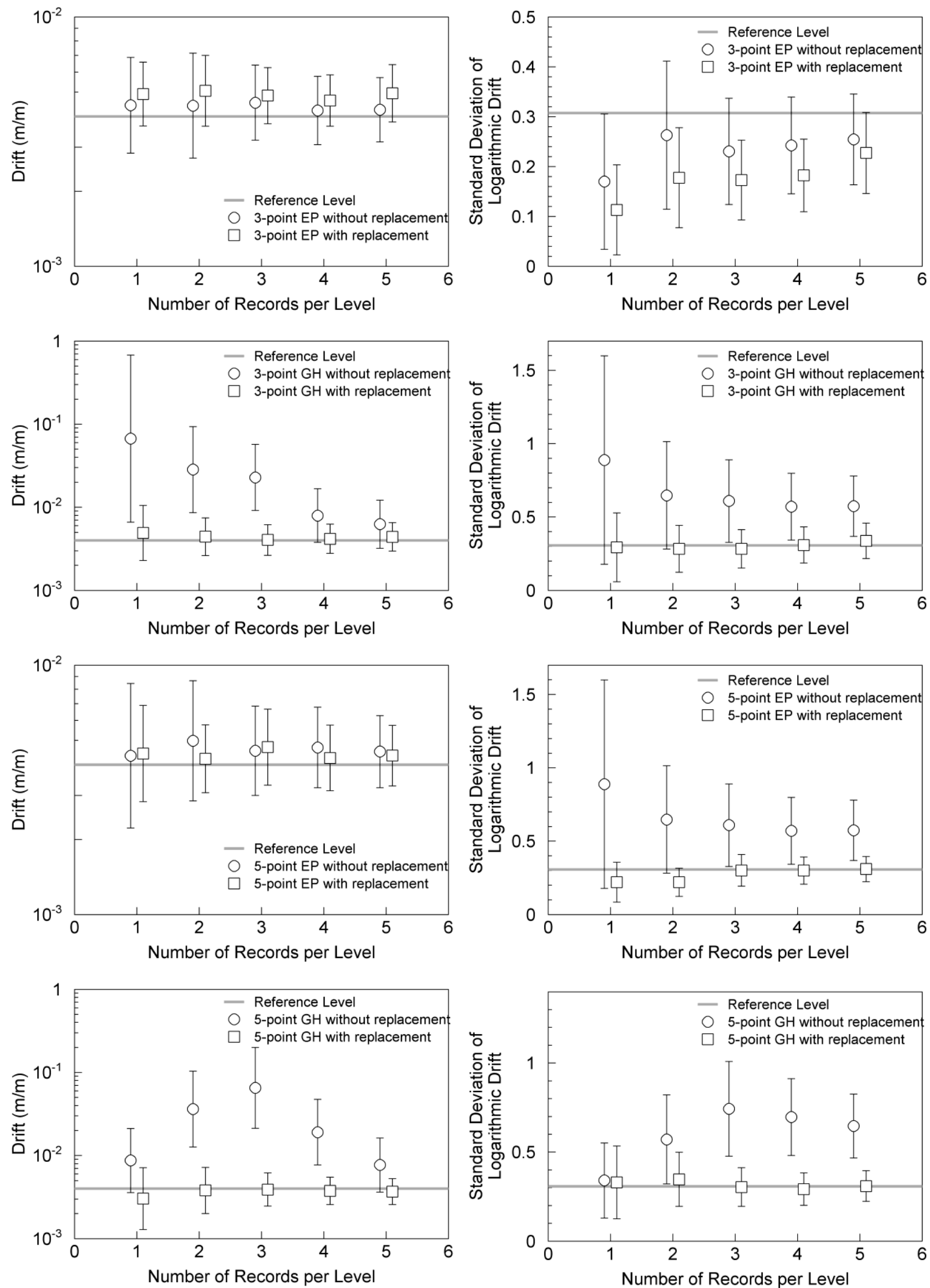

Figure 7: Results for the estimation of first-storey drift from the time-history analyses conducted on the scaled records. The panels on the left-hand-side correspond to estimates of the mean logarithmic drift while those on the right-hand-side correspond to estimates of the standard deviation of the logarithmic drifts. In all cases the error bars represent the $95 \%$ confidence intervals for the estimates. 

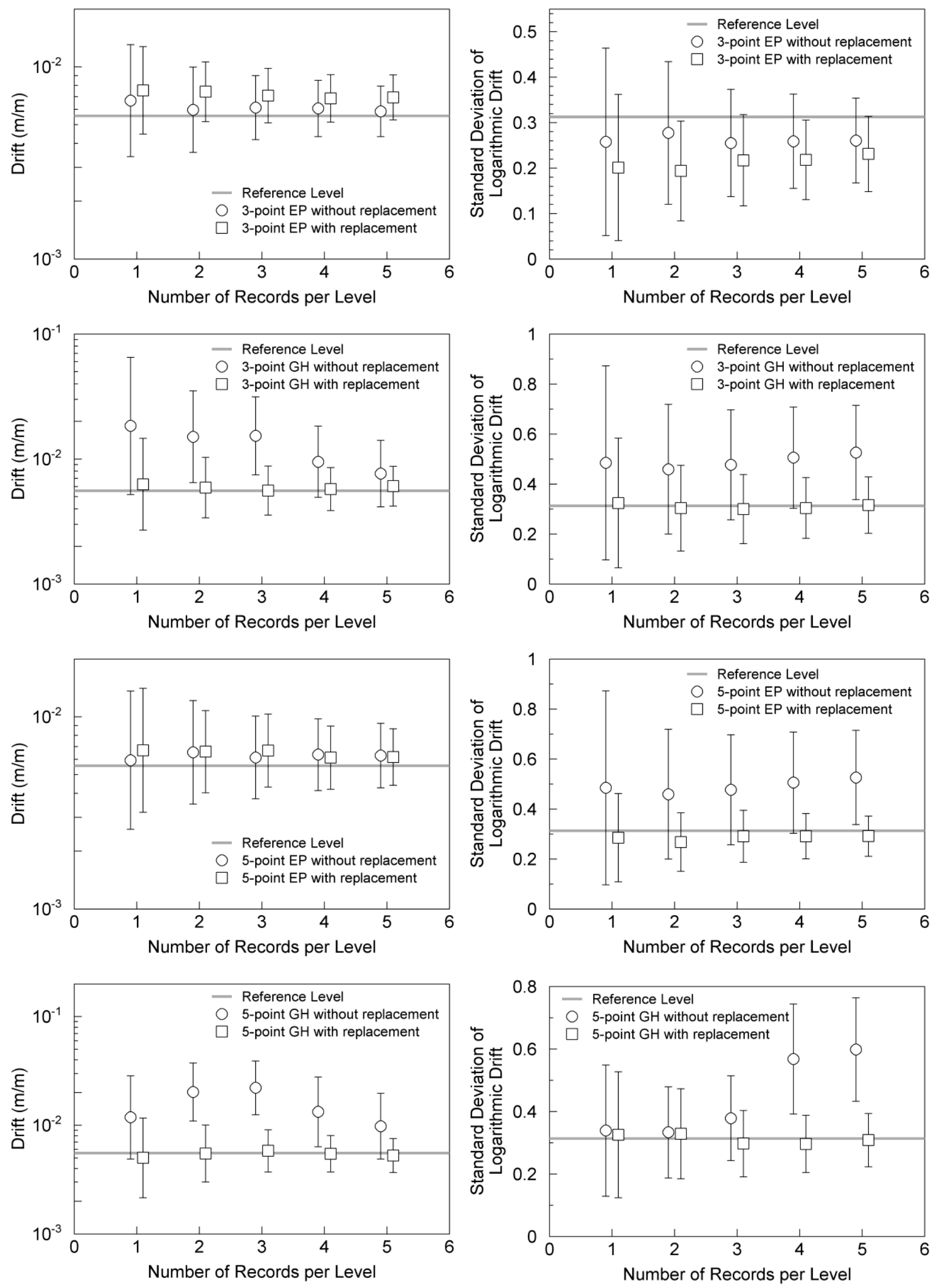

Figure 8: As for Figure 7, but showing the results for the estimation of roof drift. 
Table 4: Record selection based upon using a different record scaled to each reference spectra. The numbers in the table represent the maximum drift demands in meters, i.e., directly comparable to the pushover curves shown in Figure 4. $\mathrm{GH}=$ Gauss-Hermite, EP = Equal Probability.

\begin{tabular}{|c|c|c|c|c|c|c|}
\hline & \multicolumn{6}{|c|}{ Storey } \\
\hline Method & $1^{\text {st }}$ & $2^{\text {nd }}$ & $3^{\text {rd }}$ & $4^{\text {th }}$ & $5^{\text {th }}$ & $6^{\text {th }}$ \\
\hline GH 3 levels & 0.133 & 0.111 & 0.111 & 0.087 & 0.183 & 0.144 \\
\hline GH 5 levels & 0.196 & 0.201 & 0.165 & 0.096 & 0.243 & 0.192 \\
\hline EP 3 levels & 0.035 & 0.03 & 0.036 & 0.033 & 0.096 & 0.066 \\
\hline EP 5 levels & 0.1155 & 0.09 & 0.078 & 0.054 & 0.153 & 0.075 \\
\hline
\end{tabular}

\section{Discussion and conclusions}

The results presented in Figures 7 and 8 seem to be very promising. When the target spectral levels are defined according to the roots of Hermite polynomials and individual records are scaled to match all of these different targets, it may be appreciated that both the 3-point and 5-point approximations work extremely well. This statement holds for both the estimates of the means and of the standard deviations of the logarithmic drifts. Good approximations to the full distribution of drift may therefore be obtained by using a single record with a spectral shape that is well matched to the specified scenario. This approximation is generally improved, but only very slightly, when a second record is used. It also appears that the use of a 5-point approximation does not provide significant advantages over a 3-point approximation which means that as few as six (two records, each scaled to three target levels) carefully designed time-history analyses may be all that is required in order to obtain a reasonable estimate of the full distribution of drift response. Of course, while we have presented the $95 \%$ confidence intervals for these estimates in Figures 7 and 8 we have not made any statement regarding how many records would be required to achieve a particular precision in these estimates of the first and second moments. However, it can be observed from these figures that for the preferred GH approach, and with replacement allowed during the record selection, there are no significant trends, which means that an estimate of the number of records that are required to achieve a certain level of precision can be obtained from consideration of the formulae for determining the standard errors of the estimates of the mean (Equation 7 ) and standard deviation (Equation 8). Equations 7 and 8 enable the numbers of records that are required in order to obtain estimates of the mean and standard deviation of the distribution of drift to within a particular precision.

$$
\begin{gathered}
\text { s.e. }\left(\mu_{\log _{10} \delta}\right)=\frac{\sigma_{\log 10 \delta}}{\sqrt{n_{\text {targets }} n_{\text {records }}}} \cong \frac{s_{\log 10 \delta}}{\sqrt{n_{\text {targets }} n_{\text {records }}}} \\
\text { s.e. }\left(\sigma_{\log 10 \delta}\right)=\frac{\sigma_{\log 10 \delta}}{\sqrt{2 n_{\text {targets }} n_{\text {records }}}} \cong \frac{s_{\log 10 \delta}}{\sqrt{2 n_{\text {targets }} n_{\text {records }}}}
\end{gathered}
$$


The approach using a single record scaled to match all target levels of the response spectrum performs very well, which is perhaps not unexpected given that the shape of the target spectra are always very similar, as discussed previously. When different records are selected to match each of the target levels separately, and the rule not allowing replacement of the selected records is invoked, the method is somewhat less efficient because of the still rather limited coverage of the global strong-motion databank. Given that the total number of available records in any magnitude-distance-site class bin is generally small, if a separate record is required for each target level of the spectrum, it is almost inevitable that for the third or fifth record, as the case may be, the match to the target spectral shape will not be as good as for the first record selected. Therefore, for as long as the number of candidate records remains rather limited it is advantageous to scale individual records to multiple target levels.

In this study the records were selected by first finding the record that provided the best match to the median target spectrum before progressively moving to target spectra more distant from the median. This approach makes some intuitive sense when the objective is to estimate the median drift response but it may be that for the purposes of modeling the full distribution greater priority could be placed upon ensuring better fits to the target levels away from the median.

This study has only been concerned with the estimation of the distribution of drift response and the proposed methodology in this case has proven to be effective for the single structure and earthquake scenario considered herein. Whether or not this approach would yield similar quality results when applied to alternative damage measures such as fatigue damage or absorbed hysteretic energy remains to be seen but this will most likely be related to the strength of the correlation between spectral acceleration and these parameters. For these damage measures it is likely that more importance would have to be placed upon ensuring that the duration and frequency contents of the selected records were consistent with the specified seismological scenario (Hancock and Bommer, 2006).

The recent study of Hancock et al. (2008) suggests that the approach presented in this study would work even more effectively if, in addition to linearly scaling the accelerograms so that their spectra matched the target spectra over a broad period range, these records were then adjusted with wavelets so that this match was further improved. Furthermore, it would seem that the chances that the proposed approach would work when applied to estimate the distributions of fatigue damage or absorbed hysteretic energy would be greatly enhanced if the wavelet adjustments were made to not only multiple target spectra but also to spectra constructed for different damping ratios. Hancock et al. (2008) have demonstrated that this approach further ensures that the duration of the selected records are consistent with the prescribed seismological scenario.

This article represents a first attempt at investigating the problem of how many records are required in order to obtain a robust estimate of the full distribution of a response parameter. The study has been conducted for a single six-storey reinforced concrete 
frame and for a single seismological scenario and the performance of the proposed approaches should be considered in this light. Clearly, further work is required for different earthquake scenarios and different structures in order to qualify and generalize the findings of this study as well as to refine the overall procedure. However, the results that have been presented herein are promising and suggest that it is conceivable for engineers to obtain stable estimates of the distribution of structural response parameters from a relatively small number of time-history analyses.

\section{Acknowledgements}

We would like to acknowledge the financial support of the Italian Department of Civil Protection (Reluis 2005 Grant - Task 7: Innovative techniques for seismic protection) that enabled the first author to complete this work whilst visiting Imperial College London. The second author is a fellow of the Willis Research Network and would like to acknowledge their generous financial support. We would like to acknowledge the guest editor, Dr Erol Kalkan, and two anonymous reviewers for their thorough reviews that led to a significant improvement in the manuscript.

\section{Notation}

The following symbols are used in this paper:

$\mathrm{a}, \mathrm{b} \quad=$ indices for period range over which spectral matching is evaluated

$\mathrm{C}_{\mathrm{i}} \quad=$ regression coefficients

$D_{r m s}=$ Root-mean-square difference between a scaled spectrum and a target spectrum

$F_{E}(\varepsilon)=$ cumulative distribution function of epsilon

$H_{n}(x)=n^{\text {th }}$-order Hermite polynomial

$\mathrm{M}_{\mathrm{w}} \quad=$ Moment magnitude

$\mathrm{n} \quad=$ order of Hermite polynomial

$\mathrm{n}_{\text {records }}=$ number of records at each target level

$n_{\text {targets }}=$ number of target levels

$\mathrm{p}_{\mathrm{i}} \quad=$ Gauss-Hermite quadrature weight

$\mathrm{R}_{\mathrm{JB}} \quad=$ Joyner-Boore distance

$\mathrm{S}_{\log 10 \delta}=$ sample standard deviation of logarithmic drift values

$\mathrm{S}_{\mathrm{a}}\left(\mathrm{T}_{1}\right)=$ Spectral acceleration at the initial fundamental period of the structure

$\mathrm{Sa}_{\mathrm{R}}\left(\mathrm{T}_{\mathrm{i}}\right)=$ Spectral acceleration of a record evaluated at $T_{i}$

$\mathrm{Sa}_{\mathrm{T}}\left(\mathrm{T}_{\mathrm{i}}\right)=$ Spectral acceleration of a target spectrum evaluated at $\mathrm{T}_{\mathrm{i}}$

s.e. $=$ standard error

$\mathrm{T}_{1} \quad=$ Initial fundamental period of the structure

$T_{i} \quad=i^{\text {th }}$ response period

$\mathrm{V}_{\mathrm{S}, 30}=$ Average shear-wave velocity over the upper $30 \mathrm{~m}$

$\mathrm{y} \quad=$ dependent variable

$\left\langle x^{k}\right\rangle=$ The $k^{\text {th }}$ moment of $\mathrm{x}$

$\alpha \quad=$ scaling factor

$\delta_{\mathrm{i}} \quad=$ inter-storey drift for the $\mathrm{i}^{\text {th }}$ storey 
$\varepsilon \quad=$ epsilon; the difference between observed and modelled ground motions or response in units of the standard deviation of the considered measure

$\varepsilon_{\mathrm{A}} \quad=$ normalized intra-event residual

$\varepsilon_{\mathrm{E}} \quad=$ normalized inter-event residual

$\mu_{\log 10 \square}=$ mean of the logarithmic drift values

$\sigma_{\mathrm{A}} \quad=$ intra-event variability

$\sigma_{\mathrm{C}} \quad=$ inter-component variability

$\sigma_{\mathrm{E}} \quad=$ inter-event variability

$\sigma_{\mathrm{T}} \quad=$ total variability

$\sigma_{\log 10 \delta}=$ population standard deviation of logarithmic drift values

\section{References}

Anderson, T. W., and Darling, D. A. (1952). "Asymptotic theory of certain "goodness of fit" criteria based on stochastic processes." Annals of Mathematical Statistics, 23(2), 193-212.

Baker, J. W., and Cornell, C. A. (2006a). "Spectral shape, epsilon and record selection." Earthquake Engineering and Structural Dynamics, 35(9), 1077-1095.

Baker, J. W., and Cornell, C. A. (2006b). "Which spectral acceleration are you using?" Earthquake Spectra, 22(2), 293-312.

Baker, J. W. (2007). "Probabilistic structural response assessment using vector-valued intensity measures." Earthquake Engineering and Structural Dynamics 36(13), 1861-1883.

Beyer, K., and Bommer, J. J. (2007). "Selection and scaling of real accelerograms for bidirectional loading: a review of current practice and code provisions." Journal of Earthquake Engineering, 11(S1), 13-45.

Bommer, J. J., and Acevedo, A. B. (2004). "The use of real earthquake accelerograms as input to dynamic analysis." Journal of Earthquake Engineering, 8(Special Issue 1), 43-91.

Bommer, J. J., Stafford, P. J., Alarcón, J. E., and Akkar, S. (2007). "The influence of magnitude range on empirical ground-motion prediction." Bulletin of the Seismological Society of America, 97(6), 2152-2170.

Boore, D. M., and Bommer, J. J. (2005). "Processing of strong-motion accelerograms: needs, options and consequences." Soil Dynamics and Earthquake Engineering, 25(2), 93-115.

Boore, D. M., Joyner, W. B., and Fumal, T. E. (1997). "Equations for estimating horizontal response spectra and peak ground acceleration from western North America earthquakes: a summary of recent work." Seismological Research Letters, 68(1), 128-153, Erratum, 76, 368-369.

Boore, D. M. (2003). "Simulation of ground motion using the stochastic method." Pure and Applied Geophysics, 160, 635-676.

Brady, A. G., Trifunac, M. D., and Hudson, D. E. (1973). "Analyses of strong motion earthquake accelerograms - response spectra." Technical Report, Earthquake Engineering Research Laboratory - California Institute of Technology. 
Ceb-Fip (1993). Model Code 1990, Redwood Books, Trowbridge.

Cornell, C. A., Jalayer, F., Hamburger, R. O., and Foutch, D. A. (2002). "Probabilistic basis for 2000 SAC federal emergency management agency steel moment frame guidelines." Journal of Structural Engineering, 128(4), 526 - 533.

Cornell, C. A. (2005). "On earthquake record selection for nonlinear dynamic analysis." Proceedings of the Esteva Symposium, http://eventos.iingen.unam.mx/SimposioLE/Documentos/Cornell.pdf

Gasparini, D. A., and Vanmarcke, E. H. (1979). "Simulated earthquake motions compatible with prescribed response spectra." Evaluation of Seismic Safety of Buildings Report No. 2, Department of Civil Engineering, MIT, Cambridge, Massachusetts, 99 pp.

Hancock, J., and Bommer, J. J. (2006). "A state-of-knowledge review of the influence of strong-motion duration on structural damage." Earthquake Spectra, 22(3), 827-845.

Hancock, J., Watson-Lamprey, J., Abrahamson, N. A., Bommer, J. J., Markatis, A., McCoy, E., and Mendis, R. (2006). "An improved method of matching response spectra of recorded earthquake ground motion using wavelets." Journal of Earthquake Engineering, 10(S1), 67-89.

Hancock, J., and Bommer, J. J. (2007). "Using spectral matched records to explore the influence of strong-motion duration on inelastic structural response." Soil Dynamics and Earthquake Engineering, 27(4), 291-299.

Hancock, J., Bommer, J. J., and Stafford, P. J. (2008). "Numbers of scaled and matched accelerograms required for inelastic dynamic analyses." Earthquake Engineering and Structural Dynamics, 37(13), 1585-1607.

lervolino, I., and Cornell, C. A. (2005). "Record selection for nonlinear seismic analysis of structures." Earthquake Spectra 21(3). 685-713.

Jayaram, N., and Baker, J. W. (2008). "Statistical tests of the joint distribution of spectral acceleration values." Bulletin of the Seismological Society of America 98(5), 22312243.

Joyner, W. B., and Boore, D. M. (1981). "Peak horizontal acceleration and velocity from strong-motion records including records from the 1979 Imperial valley, California, earthquake." Bulletin of the Seismological Society of America, 71(6), 2011-2038.

Keefer, D. L., and Bodily, S. E. (1983). "Three-point approximations for continuous random variables." Management Science, 29(5), 595-609.

Kottke, A., and Rathje, E. M. (2008). "A semi-automated procedure for selecting and scaling recorded earthquake motions for dynamic analysis." Earthquake Spectra 24(4), 911-932.

Lilliefors, H. W. (1967). "On the Kolmogorov-Smirnov test for normality with mean and variance unknown." Journal of the American Statistical Association, 62(318), 399402.

Lindstrom, M. J., and Bates, D. M. (1990). "Nonlinear mixed effects models for repeated measures data." Biometrics, 46, 673-687.

Luco, N., and Bazzurro, P. (2007). "Does amplitude scaling of ground motion records result in biased nonlinear structural drift responses?" Earthquake Engineering and Structural Dynamics, 36(13), 1813-1835.

Mander, J. B., Priestley, M. J. N., and Park, R. (1988). "Theoretical stress-strain model for confined concrete." Journal of Structural Engineering, 114(8), 1804-1826. 
Massey, F. J. (1951). "The Kolmogorov-Smirnov test for goodness of fit." Journal of the American Statistical Association, 46(253), 68-78.

McKenna, F., Fenves, G. L., and Scott, M. H. (2000). "Open system for earthquake engineering simulation (OpenSees)." Univ. of California, Berkeley, CA.

Menegotto, M., and Pinto, P. E. "Method for analysis for cyclically loaded R.C. plane frames including changes in geometry and non-elastic behavior of elements under combined normal force and bending." Symposium on the resistance and ultimate deformability of structures acted on by well defined repeated loads, Zurich, Switzerland, 15-22.

Miller, A. C., and Rice, T. R. (1983). "Discrete approximations for probability distributions." Management Science, 29(3), 352-362.

Naeim, F., Alimoradi, A., and Pezeshk, S. (2004). "Selection and scaling of ground motion time histories for structural design using genetic algorithms." Earthquake Spectra 20(2), 413-426.

Pinheiro, J., Bates, D., DebRoy, S., Sarkar, D., and the R Core team (2008). "nlme: linear and nonlinear mixed effects models." R package version 3.1-87.

Power, M., Chiou, B., Abrahamson, N. A., and Roblee, C. "The 'Next Generation of Ground Motion Attenuation Models' (NGA) project: an overview." Proceedings of the 8th U.S. National Conference on Earthquake Engineering, San Francisco, CA, Paper No. 2022.

Priestley, M. J. N., Calvi, G. M., and Kowalsky, M. J. (2007). "Displacement-based seismic design of structures." IUSS Press, $720 \mathrm{pp}$.

$\mathrm{R}$ Development Core Team (2008). "R: A language and environment for statistical computing." R Foundation for Statistical Computing, Vienna, Austria. ISBN 3900051-07-0, URL http://www.R-project.org.

Shapiro, S. S., and Wilk, M. B. (1965). "An analysis of variance test for normality (complete samples)." Biometrika, 52(3/4), 591-611.

Shome, N., Cornell, C. A., Bazzurro, P., and Carballo, J. E. (1998). "Earthquakes, records, and nonlinear response." Earthquake Spectra, 14(3), 469-500.

Spacone, E., Ciampi, V., and Filippou, F. C. (1996). "Mixed formulation of nonlinear beam finite element." Computers and Structures, 58(1), 71-83.

Spiegel, M. R., and Liu, J. (1999). "Mathematical handbook of formulas and tables" Schaum's Outline Series, McGraw-Hill, New York, pp. 278.

Stafford, P. J., Strasser, F. O., and Bommer, J. J. (2008). "An evaluation of the applicability of the NGA models to ground-motion prediction in the Euro-Mediterranean region." Bulletin of Earthquake Engineering, 6(2), 149-177.

Tothong, P., and Cornell, C. A. (2008). "Structural performance assessment under nearsource pulse-like ground motions using advanced ground motion intensity measures." Earthquake Engineering and Structural Dynamics 37(7), 1013-1037.

Watson-Lamprey, J., and Abrahamson, N. (2006). "Selection of time series for analyses of response of buildings." Proceedings of the First European Conference on Earthquake Engineering and Seismology, Geneva, Switzerland, paper no. 248. 